\title{
LOCATION AND SCALE BEHAVIOUR OF THE QUANTILES OF A NATURAL EXPONENTIAL FAMILY
}

\author{
Mauro Piccioni ${ }^{1}$, Bartosz KołodziejeK ${ }^{2}$ and GÉrard LetaC ${ }^{3, *}$
}

\begin{abstract}
Let $P_{0}$ be a probability on the real line generating a natural exponential family $\left(P_{t}\right)_{t \in \mathbb{R}}$. Fix $\alpha$ in $(0,1)$. We show that the property that $P_{t}((-\infty, t)) \leq \alpha \leq P_{t}((-\infty, t])$ for all $t$ implies that there exists a number $\mu_{\alpha}$ such that $P_{0}$ is the Gaussian distribution $N\left(\mu_{\alpha}, 1\right)$. In other terms, if for all $t$, the number $t$ is a quantile of $P_{t}$ associated to some threshold $\alpha \in(0,1)$, then the exponential family must be Gaussian. The case $\alpha=1 / 2$, i.e. when $t$ is always a median of $P_{t}$, has been considered in Letac et al. [Statist. Prob. Lett. 133 (2018) 38-41]. Analogously let $Q$ be a measure on $[0, \infty)$ generating a natural exponential family $\left(Q_{-t}\right)_{t>0}$. We show that $Q_{-t}\left(\left[0, t^{-1}\right)\right) \leq \alpha \leq Q_{-t}\left(\left[0, t^{-1}\right]\right)$ for all $t>0$ implies that there exists a number $p=p_{\alpha}>0$ such that $Q(\mathrm{~d} x) \propto x^{p-1} \mathrm{~d} x$, and thus $Q_{-t}$ has to be a gamma law with parameters $p$ and $t$.
\end{abstract}

Mathematics Subject Classification. 62E10, 60E05, 45E10.

Received February 4, 2019. Accepted May 16, 2019.

\section{INTRODUCTION}

Let $P_{0}$ be a probability on the real line and assume that its moment generating function

$$
M(t)=\int_{-\infty}^{+\infty} e^{t x} P_{0}(\mathrm{~d} x)
$$

is finite for all real $t$. Such a probability generates the natural exponential family

$$
P_{t}(\mathrm{~d} x)=\frac{e^{t x}}{M(t)} P_{0}(\mathrm{~d} x), t \in \mathbb{R}
$$

parametrized by the natural parameter $t$.

For example, the Gaussian probability $P_{0}(\mathrm{~d} x)=\left(2 \pi \sigma^{2}\right)^{-1 / 2} e^{-(x-m)^{2} / 2 \sigma^{2}}$ i.e. $P_{0}=N\left(m, \sigma^{2}\right)$, with $m \in \mathbb{R}$ and $\sigma^{2}>0$, generates the natural exponential family $\left(P_{t}\right)=\left(N\left(m+t \sigma^{2}, \sigma^{2}\right)\right)$. In this case, if $X_{t} \sim P_{t}$ for any

Keywords and phrases: Characterization of normal and gamma laws, one-dimensional exponential families, quantiles of a distribution, Deny equations.

1 Dipartimento di Matematica, Sapienza Università di Roma, 00185 Roma, Italy.

2 Faculty of Mathematics and Information Sciences, Warsaw University of Technology, Koszykowa 75, 00-662 Warsaw, Poland.

${ }^{3}$ Laboratoire de Statistique et Probabilités, Université Paul Sabatier, 118 Route de Narbonne, 31062 Toulouse, France.

* Corresponding author: gerard.letac@math.univ-toulouse.fr 
$t \in \mathbb{R}$, then $X_{t} \sim X_{0}+\sigma^{2} t$. In other words $\left(P_{t}\right)$ is a location family generated by $P_{0}$ with location parameter $\sigma^{2} t$. It is easily verified that the property $X_{t} \sim X_{0}+\sigma^{2} t$ forces a natural exponential family to be generated by $P_{0}=N\left(m, \sigma^{2}\right)$ for some $m$. A way to see this is to compute the moment generating function of $X_{t}$ and substitute $X_{0}+\sigma^{2} t$ to $X_{t}$, getting the equation

$$
M(t+s)=M(t) M(s) e^{\sigma^{2} t s}, \quad t, s \in \mathbb{R} .
$$

Taking $\operatorname{logs}$ and deriving with respect to $t$ and $s$ we get that the cumulant generating function $k=\log M$ of $P_{0}$ satisfies $k^{\prime \prime}(u)=\sigma^{2}$ for all $u \in \mathbb{R}$, from which $k(u)=-m u+u^{2} / 2 \sigma^{2}$, that is precisely the cumulant generating function of $N\left(m, \sigma^{2}\right)$.

A complete characterization of the general exponential families which are location families has been given in [2]. However, if we restrict our attention to natural exponential families only the Gaussian families mentioned remain.

The following remark is quite natural: the assumption that $X_{t} \sim X_{0}+\sigma^{2} t$, for any $t \in \mathbb{R}$, means that the distribution function of $X_{t}-\sigma^{2} t$ is independent of $t$, and so the same is true for the quantile function. If we make the weaker assumption that for some fixed $\alpha \in(0,1)$ an $\alpha$-quantile of $X_{t}-\sigma^{2} t$ does not depend on $t$, does one obtain the same characterization established above? In slightly simplified words, if $X_{t} \sim P_{t}$, as defined in (1.2), is such that $\operatorname{Pr}\left(X_{t} \leq \sigma^{2} t+b\right)=\alpha$ for any $t \in \mathbb{R}$, for some fixed $b \in \mathbb{R}$, does this imply that $P_{0}$ is $N\left(m, \sigma^{2}\right)$ for some $m$ ?

A recent paper [3] gives the answer to this question for $\alpha=1 / 2, b=0$ and $\sigma^{2}=1$. Indeed it is proved there that if $t$ is a median of $P_{t}$, for any $t \in \mathbb{R}$, then $P_{0}$ is the standard Gaussian $N(0,1)$. The first result of the present paper is the extension of this result for any $\alpha \in(0,1)$ (and arbitrary $b$ and $\sigma^{2}$ ).

Theorem 1.1. Let $P_{0}$ be a probability on the real line which generates the exponential family (1.2). Let $b \in \mathbb{R}$ and suppose that $b+\sigma^{2} t$ is an $\alpha$-quantile of $P_{t}$, for $t \in \mathbb{R}$, that is

$$
\int_{\left(-\infty, b+\sigma^{2} t\right)} e^{t x} P_{0}(\mathrm{~d} x) \leq \alpha M(t) \leq \int_{\left(-\infty, b+\sigma^{2} t\right]} e^{t x} P_{0}(\mathrm{~d} x), \quad t \in \mathbb{R}
$$

Then $P_{0}=N\left(m^{*}, \sigma^{2}\right)$, where $m^{*}=b-\sigma \Phi^{-1}(\alpha)$, $\Phi$ being the standard Gaussian distribution function; moreover $P_{t}=N\left(m^{*}+\sigma^{2} t, \sigma^{2}\right)$.

For proving Theorem 1.1 we will make use (see (2.3)) of a function proportional to $\alpha x_{-}+(1-\alpha) x_{+}$(where $x_{+}=\max (0, x)$ and $\left.x_{-}=(-x)_{+}\right)$. Notice that

$$
t \mapsto \mathbb{E}\left(\alpha(Z-t)_{-}+(1-\alpha)(Z-t)_{+}\right)
$$

obtains its minimum on the $\alpha$-quantile of the integrable random variable $Z$. This last fact implies that the empirical $\alpha$-quantiles are $M$-estimators (for a definition, see [5], p. 41).

We describe now our second result: Let $Q$ be a Radon measure on the non-negative real half-line such that its Laplace transform

$$
L(t)=\int_{[0,+\infty)} e^{-t y} Q(\mathrm{~d} y)
$$

is finite for all $t>0$. Such a measure generates the exponential family

$$
Q_{-t}(\mathrm{~d} y)=\frac{e^{-t y}}{L(t)} Q(\mathrm{~d} y), t>0
$$


parametrized by the natural parameter $t$ (notice the change of sign w.r.t. the standard usage). In principle this family could be smaller than the natural exponential family generated by $Q$, but it will turn out not to be the case. As an example, the measure

$$
Q^{p}(\mathrm{~d} y)=\frac{1}{\Gamma(p)} y^{p-1} \mathrm{~d} y
$$

defined for $p>0$ generates the natural exponential family $Q_{-t}^{p}=\mathrm{Ga}(p, t)$, with $t>0$, where $\mathrm{Ga}(p, t)$ is the gamma law with parameters $p$ and $t$. Now it is immediately verified that if $Y_{t} \sim Q_{-t}^{p}$ then $Y_{t} \sim Y_{1} / t$, that is $\left(Q_{-t}^{p}\right)$ is a scale family generated by $Q_{-1}^{p}=\mathrm{Ga}(p, 1)$, with scale parameter $t^{-1}$. It is relatively easy to verify that this property forces $Q$ to be of the form (1.6). However the argument in the scale case is slightly more involved than in the location case and we prefer to give the statement as a proposition.

Proposition 1.2. Suppose that $\left(Q_{-t}\right)_{t>0}$ is the natural exponential family defined in (1.5), for some measure $Q$ on the non-negative real half-line. With $Y_{t} \sim Q_{-t}$, assume that $Y_{t} \sim \frac{Y_{1}}{t}$ for any $t>0$. Then, up to a multiplicative constant, $Q=Q^{p}$ defined by (1.6), for some $p>0$.

Proof. Proof of Proposition 1.2 Compute the Laplace transform of $Y_{t}$ in the point $s t$, where $s, t>0$. Then using the assumption $Y_{t} \sim \frac{Y_{1}}{t}$ one arrives at

$$
\frac{L(t+t s)}{L(t)}=\frac{L(1+s)}{L(1)}
$$

Defining $c(t)=\log L(t)$, for $t>0$ and deriving w.r.t. $t$ and $s$ this implies

$$
u c^{\prime \prime}(u)+c^{\prime}(u)=0
$$

where $u=t+t s>0$. Integrating twice one arrives at $c(u)=-p \log u+\ell$, with $p>0$ and an arbitrary $\ell \in \mathbb{R}$, from which $L(u)=e^{\ell} / u^{p}$, the Laplace transform of $e^{\ell} Q^{p}$.

Again the statement of the previous proposition is a special case of the general result contained in [2], where all the general exponential families which are scale families are determined. However, only the above ones are natural exponential families.

The assumption $Y_{t} \sim Y_{1} / t$ for any $t>0$ is equivalent to say that the distribution function of $t Y_{t}$ is independent of $t$, and so the same is true for the quantile function. If we make the weaker assumption that, for some fixed $\alpha \in(0,1)$, an $\alpha$-quantile of $t Y_{t}$ does not depend on $t$, is it enough to obtain the characterization stated in Proposition 1.2? In slightly simplified words, if $Y_{t} \sim Q_{-t}$ as defined in (1.5), is such that $\operatorname{Pr}\left(Y_{t} \leq a / t\right)=\alpha$ for all $t>0$, for some $a>0$, does this still imply that $Q$ is proportional to $Q^{p}$ for some $p>0$ ? Our second result gives a positive answer to this conjecture.

Theorem 1.3. Let $Q$ be a Radon measure on the non-negative real half-line which generates the exponential family (1.5). Let $a>0$ and suppose that $a / t$ is an $\alpha$-quantile of $Q_{-t}$, for $t>0$, that is

$$
\int_{(0, a / t)} e^{-t y} Q(\mathrm{~d} y) \leq \alpha L(t) \leq \int_{(0, a / t]} e^{-t y} Q(\mathrm{~d} y), \quad t>0 .
$$

Then $Q$ is proportional to $Q^{p^{*}}$, where $p^{*}=p^{*}(\alpha)$ is the unique solution in $p>0$ of the equation $E_{p}(a)=\alpha, E_{p}$ being the distribution function of $G a(p, 1)$. In addition $Q_{-t}=G a\left(p^{*}, t / a\right)$.

It is convenient to comment on the existence and the uniqueness of $p^{*}$. The family $(\mathrm{Ga}(p, 1), p>0)$ is a convolution semigroup of laws supported by $[0, \infty)$. Hence, for any fixed $a>0$ the function $p \mapsto E_{p}(a)$ is strictly 
decreasing in $p$ and is continuous. From the Markov inequality and the fact that the expectation of $\operatorname{Ga}(p, 1)$ is

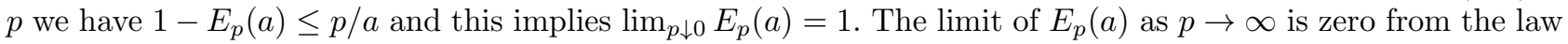
of large numbers.

The proofs of Theorems 1.1 and 1.3 are given in the next sections. These proofs deduce from (1.3) and (1.7) two convolution equations of type

$$
f=f * H
$$

in additive and multiplicative forms, respectively. The solutions to these equations have been investigated in [1]. The result for additive convolutions is reported in the final section of [1]. The result for multiplicative convolutions can be obtained with a passage to the additive convolution form by taking logarithms. In the next proposition we report both of them explicitly.

Proposition 1.4. (1) Suppose $H$ is a probability density on $\mathbb{R}$, and consider the equation

$$
f(t)=\int_{-\infty}^{+\infty} H(t-x) f(x) \mathrm{d} x, \quad t \in \mathbb{R}
$$

where $f$ is a locally integrable, non-negative function. Then $f$ is necessarily a linear combination, with non-negative coefficients, of a constant function with an exponential function of the form $e^{-s^{*} x}$, where $s^{*} \neq 0$ is a solution of the following equation in the real unknown $s$

$$
\int_{-\infty}^{+\infty} e^{s x} H(x) \mathrm{d} x=1
$$

If there is no solution of this form then $f$ is necessarily constant.

(2) Suppose $K$ is a probability density on the positive real half-line and consider the equation

$$
g(t)=\int_{0}^{+\infty} K\left(\frac{t}{y}\right) g(y) \frac{\mathrm{d} y}{y}, \quad t>0
$$

where $g$ is a locally integrable and non-negative function on $(0, \infty)$. Then $g(t)$ is necessarily a linear combination, with non-negative coefficients, of the function $t^{-1}$ with a power function of the form $t^{-1-u^{*}}$, where $u^{*} \neq 0$ is a solution of the following equation in the real unknown $u$

$$
\int_{0}^{+\infty} y^{u} K(y) \mathrm{d} y=1
$$

If there is no solution of this form then $g(t)=c / t$, where $c \geq 0$.

Both the equations (1.9) and (1.11) have at most one non zero solution in $s$ and $u$, respectively. Indeed, the logarithm of the l.h.s of of these equations are convex functions of $s$ and $u$ respectively.

A last section mentions that such a characterization of the normal distribution could be used to design a test of Gaussianity based only on a fixed quantile, and that a similar test could be done for a gamma distribution.

\section{Proof of Theorem 1.1}

We notice that it is enough to prove the result with $\sigma^{2}=1$. Indeed, it easily proved that if $\left(P_{t}, t \in \mathbb{R}\right)$ is a natural exponential family and $X_{t} \sim P_{t}$, then, for any $\sigma>0$ the family $\left(P_{t}^{\prime}, t^{\prime} \in \mathbb{R}\right)$, where $\sigma^{-1} X_{t / \sigma} \sim P_{t}^{\prime}$, is another natural exponential family with natural parameter $t$. Moreover if $\left(P_{t}\right)$ is a location family with location parameter $\sigma^{2} t$, then $\left(P_{t}^{\prime}\right)$ is a location family with location parameter $t$. 
Let us prove the theorem with $b=0$. Then we will adjust the solution to take into account an arbitrary value of $b$. First we prove that $P_{0}$ is absolutely continuous. Take $-A \leq s<t \leq A$, for some constant $A>0$ and compute

$$
\begin{gathered}
P_{0}((s, t))=\int_{(s, t)} e^{-t x} e^{t x} P_{0}(\mathrm{~d} x) \leq e^{A^{2}} \int_{(s, t)} e^{t x} P_{0}(\mathrm{~d} x) \\
\leq e^{A^{2}}\left(\int_{(-\infty, t)} e^{t x} P_{0}(\mathrm{~d} x)-\int_{(-\infty, s]} e^{s x} P_{0}(\mathrm{~d} x)+\int_{(-\infty, s]}\left(e^{s x}-e^{t x}\right) P_{0}(\mathrm{~d} x)\right) .
\end{gathered}
$$

Using (1.3) and the inequality $\left|e^{u}-e^{v}\right| \leq|u-v| e^{w}$, for $|u|,|v| \leq w$, this is bounded by

$$
e^{A^{2}}\left(\alpha(M(t)-M(s))+|t-s| \int_{\mathbb{R}}|x| e^{A|x|} P_{0}(\mathrm{~d} x)\right) \leq c_{A}|t-s|
$$

since $M$, being analytic, is locally Lipschitz, and the integral at the l.h.s. is finite by the existence of the moment generating function of $P_{0}$ on $\mathbb{R}$.

So we can assume that $P_{0}$ has a density $p_{0}$. Setting $\alpha=\frac{C}{1+C}$, with $C>0$, the quantile relation (1.3) leads to

$$
\int_{-\infty}^{t} e^{t x} p_{0}(x) \mathrm{d} x=C \int_{t}^{+\infty} e^{t x} p_{0}(x) \mathrm{d} x .
$$

Differentiating w.r.t. $t$ both sides and multiplying by $e^{-t^{2}}$ one gets

$$
p_{0}(t)+e^{-t^{2}} \int_{-\infty}^{t} x e^{t x} p_{0}(x) \mathrm{d} x=-C p_{0}(t)+C e^{-t^{2}} \int_{t}^{+\infty} x e^{t x} p_{0}(x) \mathrm{d} x .
$$

Introduce the function defined by

$$
\operatorname{abs}_{C}(x)=-C x 1_{\{x<0\}}+x 1_{\{x>0\}} .
$$

Multiply both sides of (2.1) by $t e^{-t^{2}}$ and subtract from (2.2). We obtain

$$
p_{0}(t)=\frac{1}{1+C} \int_{-\infty}^{+\infty} \operatorname{abs}_{C}(t-x) e^{t(x-t)} p_{0}(x) \mathrm{d} x .
$$

As expected, a solution to the equation (2.4) is given by $\varphi\left(t-m^{*}\right)$, where $\varphi$ is the standard Gaussian density function, and $m^{*}=-\Phi^{-1}(\alpha)$. Next set

$$
p_{0}(x)=\varphi(x-m) f(x)
$$

with $m=m^{*}$. We aim to prove that $f(x)$ has to be constant to solve the equation (2.4), with the substitution (2.5). Rewriting the equation for $f$, one gets

$$
f(t) e^{m t-t^{2} / 2}=\frac{1}{1+C} \int_{-\infty}^{+\infty} \operatorname{abs}_{C}(t-x) e^{t(x-t)+m x-x^{2} / 2} f(x) \mathrm{d} x
$$

which is equivalent to

$$
f(t)=\frac{e^{\frac{m^{2}}{2}}}{1+C} \int_{-\infty}^{+\infty} \operatorname{abs}_{C}(t-x) e^{-\frac{(t-x+m)^{2}}{2}} f(x) \mathrm{d} x
$$


which has the form (1.8) with

$$
H(x)=\frac{e^{\frac{m^{2}}{2}}}{1+C} \operatorname{abs}_{C}(x) e^{-\frac{(x+m)^{2}}{2}} .
$$

The moment generating function of $H$ can be exactly computed

$$
\int_{-\infty}^{+\infty} e^{s x} H(x) \mathrm{d} x=1+\sqrt{2 \pi} e^{(s-m)^{2} / 2}(s-m)(\Phi(s-m)-\alpha)
$$

This is clearly equal to 1 only if $s=0$ (hence $H$ is a density) and if $s=m$. We apply Proposition $1.4,1$ ) to the equation (2.6). When $m=0$, that is if $\alpha=\frac{1}{2}$, the r.h.s. of (2.8) is equal to 1 only in 0 , hence the only non-negative non trivial solutions of the convolution equation (1.8) with kernel $H$ given by (2.7) are the positive constants. This yields immediately that $p_{0}(x)=\varphi(x)$, as desired. In the case $\alpha \neq \frac{1}{2}$ the solutions $f(x)$ are linear combinations with non-negative coefficients of the constant 1 and the function $e^{-m x}$. Coming back to $p_{0}(x)=f(x) \varphi(x-m)$, this gives density solutions for $p_{0}$ which are mixtures of $N(m, 1)$ with $N(0,1)$. But only the first one has the distribution function at 0 equal to $\alpha \neq 1 / 2$, therefore a positive component from $N(0,1)$ is forbidden. This proves that $p_{0}(x)$ has to be $\varphi(x-m)$.

Finally, to deal with an arbitrary value for $b$, define $\tau_{-b}(x)=x-b$. Now observe that if $P_{t}$ has $\alpha$-quantile $b+t$ then $P_{t}^{*}=P_{t} \circ \tau_{-b}^{-1}$ has $\alpha$-quantile $t$ and it is still a natural exponential family, for $t \in \mathbb{R}$. So $P_{t}^{*}=N\left(-\Phi^{-1}(\alpha), t\right)$ and $P_{t}=N\left(-\Phi^{-1}(\alpha)+b+t, 1\right)$, ending the proof of Theorem 1.1.

\section{Proof of Theorem 1.3}

First we prove the result with $a=1$. Assume the relation (1.7) and let $t \rightarrow 0+$. From the inequality

$$
\int_{0}^{1 / t} e^{-x t} Q(\mathrm{~d} x) \leq \alpha \int_{0}^{\infty} e^{-x t} Q(\mathrm{~d} x)
$$

where $0<\alpha<1$ we see easily that $A=Q\left(\mathbb{R}^{+}\right)$cannot be finite: for if $A$ was finite the dominated convergence for $f_{n}(x)=e^{-x / n} 1_{[0, n]}(x) \leq 1$ would lead to the contradiction $A \leq \alpha A$. Hence the natural parameter space of the natural exponential family $\left(Q_{s}\right)$ coincides with the negative reals.

Next we prove that $Q$ is absolutely continuous. Take $0<s<t<+\infty$ and compute

$$
\begin{gathered}
Q\left(\left(t^{-1}, s^{-1}\right)\right)=\int_{\left(t^{-1}, s^{-1}\right)} e^{s x} e^{-s x} Q(\mathrm{~d} x) \leq e \int_{\left(t^{-1}, s^{-1}\right)} e^{-s x} Q(\mathrm{~d} x) \\
=e\left(\int_{\left(0, s^{-1}\right)} e^{-s x} Q(\mathrm{~d} x)-\int_{\left(0, t^{-1}\right]} e^{-t x} Q(\mathrm{~d} x)+\int_{\left(0, t^{-1}\right]}\left(e^{-t x}-e^{-s x}\right) Q(\mathrm{~d} x)\right) .
\end{gathered}
$$

By (1.7) the difference between the first two integrals at the r.h.s. is bounded by $\alpha(L(s)-L(t))$, whereas the remaining integral is non positive. Again since $L$ is analytic in the positive real half-line it is locally Lipschitz and this proves the absolute continuity of $Q$, that is $Q(\mathrm{~d} x)=q(x) \mathrm{d} x$, with $q$ non-negative and locally integrable.

Now we can write (1.7) in the form of an equality, setting again $\alpha=\frac{C}{1+C}$, namely

$$
\int_{0}^{t^{-1}} e^{-t y} q(y) \mathrm{d} y=C \int_{t^{-1}}^{+\infty} e^{-t y} q(y) \mathrm{d} y, t>0 .
$$


Differentiating both sides w.r.t. $t$, one gets

$$
\frac{1+C}{t^{2}} e^{-1} q\left(t^{-1}\right)=C \int_{t^{-1}}^{+\infty} y e^{-t y} q(y) \mathrm{d} y-\int_{0}^{t^{-1}} y e^{-t y} q(y) \mathrm{d} y .
$$

Adding the 1.h.s. of (3.1) and subtracting the r.h.s., both multiplied by $t^{-1}$, to the r.h.s. of the above equality, we get for any $t>0$

$$
q\left(t^{-1}\right)=\frac{e t^{2}}{1+C}\left\{\int_{0}^{t^{-1}}\left(t^{-1}-y\right) e^{-y t} q(y) \mathrm{d} y+C \int_{t^{-1}}^{+\infty}\left(y-t^{-1}\right) e^{-y t} q(y) \mathrm{d} y\right\} .
$$

With the help of the function $\operatorname{abs}_{C}$ defined in (2.3), equality (3.2) is rewritten as

$$
q\left(t^{-1}\right)=\frac{e t}{(1+C)} \int_{0}^{\infty} \operatorname{abs}_{C}(1-t y) e^{-t y} q(y) \mathrm{d} y, t>0 .
$$

Next, for any $p>0$, define $q_{p}(x)=\frac{1}{\Gamma(p)} x^{p-1} 1_{(0,+\infty)}(x)$. Recall from the introduction that for $p=p^{*}(\alpha)$ one has

$$
\int_{0}^{1} y^{p^{*}-1} e^{-y} \mathrm{~d} y=C \int_{1}^{+\infty} y^{p^{*}-1} e^{-y} \mathrm{~d} y
$$

Now multiply both sides of (3.3) by $t^{p^{*}-2}$ and change the variable of integration at the r.h.s. to be $z=y^{-1}$. One gets

$$
t^{p^{*}-2} q\left(t^{-1}\right)=\frac{e t^{p^{*}-1}}{1+C} \int_{0}^{\infty} \operatorname{abs}_{C}\left(1-t z^{-1}\right) e^{-t / z} g\left(z^{-1}\right) \frac{d z}{z^{2}}
$$

Defining the l.h.s. of the above equality to be $g(t)$, one has

$$
q(t)=g\left(t^{-1}\right) t^{p^{*}-2}
$$

and turns the equation (3.5) into an equation of the form (1.10) in $g$ with

$$
K(y)=\frac{e}{1+C} \operatorname{abs}_{C}(1-y) e^{-y} y^{p^{*}-1} 1_{(0,+\infty)}(y) .
$$

The Mellin transform of $K$ can be easily computed

$$
\int_{0}^{\infty} y^{u} K(y) \mathrm{d} y=1+e \Gamma\left(p^{*}+u\right)\left(p^{*}+u-1\right)\left\{\alpha-E_{p^{*}+u}(1)\right\} .
$$

Now observe that the quantity inside the brackets of (3.8) at the r. h. s. is always increasing in $u$; moreover it is equal to 0 for $u=0$, due to (3.4). Hence for any value of $C>0$ the function $K$ is always a density. When $p^{*}=1$ (equivalently, $C=e-1$, or $\alpha=1-e^{-1}$ ), $u=0$ is the unique global minimum point of the r. h. s. of (3.8). Then, by Proposition 1.4, 2), the only non negative non trivial solutions to the equation (1.10), with $K$ given by (3.7), have necessarily the form $g_{0}(t)=c_{0} t^{-1}$, with $c_{0}>0$. Thus $q(y)=c_{0} q_{p^{*}}(y)=c_{0}^{\prime} y^{p^{*}-1}$. Moreover, for $p^{*} \neq 1$ (equivalently, $C \neq e-1$, or $\alpha \neq 1-e^{-1}$ ) the value $u=1-p^{*} \neq 0$ makes the expression at the $\mathrm{r}$. $\mathrm{h}$. s. of (3.8) equal to 1 , too. As a consequence $g_{1}(t)=t^{p^{*}-2}$ is also a solution of the multiplicative convolution 
equation with $K$ given by (3.7). Applying again Proposition 1.4,2) all the non negative non trivial solutions are linear combinations

$$
g_{0}(t)+c_{1} g_{1}(t)=c_{0} t^{-1}+c_{1} t^{p^{*}-2},
$$

with $c_{0}, c_{1} \geq 0$ not both zero. Substituting in (3.6) we get that the solutions to (3.2) have the form

$$
q(y)=c_{0}^{\prime} y^{p^{*}-1}+c_{1} .
$$

But for $c_{1}>0$ the condition (3.1) is violated for $t=1$. Indeed, in this case the difference between the 1.h.s. and the r.h.s. of (3.1) is equal to $c_{1}\left(1-\frac{1+C}{e}\right)$ and this is different from 0 as soon as $C \neq e-1$. So as desired $q(y)=c_{0}^{\prime} y^{p^{*}-1}$.

Finally, to deal with an arbitrary value of $a>0$, first define $\sigma_{a^{-1}}$ to be the multiplication by $a^{-1}$. Now observe that, if $Q_{-t}$ has $\alpha$-quantile $a t^{-1}$ then $Q_{-t}^{*}=Q_{-t} \circ \sigma_{a^{-1}}^{-1}$ has $\alpha$-quantile $t^{-1}$ and it is still a natural exponential family, for $t>0$. So $Q_{-t}^{*}=\mathrm{Ga}\left(p^{*}, t\right)$ and $Q_{-t}=\mathrm{Ga}\left(p^{*}, a t^{-1}\right)$, ending the proof of Theorem 1.3.

\section{Statistical CONSIDERATiOns}

A statistical application of the above characterization consists in the following exploratory testing procedure for normality. Let $X_{1}, \ldots, X_{n}$ be an i.i.d. sample from a probability measure $P_{0}$ on the real line. The obvious estimate of the $\alpha$-quantile of $P_{0}$ is the empirical $\alpha$-quantile, computed on the sample $X_{1}, \ldots, X_{n}$ and a graph of the different $\alpha$-quantiles gives an indication about the Gaussianity of $P_{0}$ : this is what a Q-Q plot does (see [6]). However, Theorem 1.1 justifies a different procedure which consists in estimating the $\alpha$-quantiles of $P_{t}$, as given by (1.2), for the same $\alpha$ and various values of $t$, and fitting to them a line.

The general principle is this: observing a sample drawn from the unknown distribution $P_{0}$, we can approximate $P_{t}(\mathrm{~d} x)=e^{t x} P_{0}(\mathrm{~d} x) / M(t)$ with the random probability measure

$$
P_{t}^{(n)}(\mathrm{d} x)=\frac{n}{\sum_{j=1}^{n} e^{t X_{j}}} \times \frac{1}{n} \sum_{i=1}^{n} e^{t X_{i}} \delta_{X_{i}},
$$

which, by the law of large numbers, converges weakly towards $P_{t}$ a.s. The $\alpha$-quantile of $P_{t}$ can be estimated with the $\alpha$-quantile $q_{t}^{(n)}$ of $P_{t}^{(n)}$ and a good fitting of a line to the points $\left(t_{i}, q_{t_{i}}^{(n)}\right)$ for some choice of $t_{i}, i=1, \ldots, m$, is an indication favouring the hypothesis that $P_{0}$ is Gaussian. In a similar fashion, the characterization of Theorem 1.3 can be used for an exploratory test of the hypothesis that a sample is drawn from a Gamma distribution: in this case the estimated $\alpha$-quantiles are plotted against the natural parameter $t$ on a log-log scale and fitted with a line of slope -1 .

Acknowledgements. B. Kołodziejek was partially supported by the grant 2016/21/B/ST1/00005 of National Science Center, Poland.

\section{REFERENCES}

[1] J. Deny, Sur l'équation de convolution $\mu=\mu * \sigma$. Séminaire Brelot-Choquet-Deny (Théorie du Potentiel) 4 (1959-1960) Talk no. $5,1-11$.

[2] T.S. Ferguson, Location and scale parameters in exponential families of distributions. Ann. Math. Statist. 33 (1962) $986-1001$.

[3] G. Letac, L. Mattner and M. Piccioni, The median of an exponential family and the normal law. Statist. Prob. Lett. 133 (2018) 38-41.

[4] D.B. Rubin, The calculation of posterior distributions by data augmentation: comment: a noniterative sampling/importance resampling alternative to the data augmentation algorithm for creating a few imputations when fractions of missing information are modest: the SIR algorithm. J. Am. Statist. Assoc. 82 (1987) 543-546.

[5] A.W. van der Vaart, Asymptotic statistics. Cambridge University Press (1998).

[6] M.B. Wilk and R. Gnanadesikan, Probability plotting methods for the analysis of data. Biometrika 55 (1968) $1-17$. 